EPJ Web of Conferences 116, 11011 (2016)

DOI: $10.1051 /$ epjconf/201611611011

(C) Owned by the authors, published by EDP Sciences, 2016

\title{
Neutrino-nucleus interactions in the few-GeV region
}

\author{
J. Nieves ${ }^{\mathrm{a}}$
}

Instituto de Física Corpuscular (IFIC), Centro Mixto CSIC-Universidad de Valencia, Institutos de Investigación de Paterna, 46071 Valencia, Spain

\begin{abstract}
Knowledge of neutrino interaction cross sections is an important and necessary ingredient in any neutrino measurement and it is crucial to achieve the precision goals of the neutrino oscillation program, making possible new discoveries like the $\mathrm{CP}$ violation in the leptonic sector. We briefly review the major features of the neutrino cross sections in the few-GeV region, paying special attention to nuclear effects.
\end{abstract}

\section{Introduction}

Recent interest in neutrino interactions in the few $\mathrm{GeV}$ energy region comes from neutrino oscillation experiments and their need to reduce systematic errors. This is because neutrinos are detected through their interactions with the nuclei that form part of the detectors. Therefore, a better understanding of weak interaction mechanisms in dense matter is actively pursued [1-3]. However, the relevance of neutrino interactions with matter extends over a large variety of topics in astrophysics, physics beyond the Standard Model, hadronic physics and nuclear physics. Thus, for instance, the interactions of electroweak probes with nuclei are interesting as a tool to discover properties of hadrons and their modification in the nuclear medium, since they penetrate deep inside the nucleus assessing the densest regions. This allows to study various aspects of the many-body problem such as nuclear correlations, meson exchange currents and relativistic effects. At intermediate energies, it is also possible to investigate the excitation of baryonic resonances and their in-medium modifications. Moreover, the medium effects in these processes can be linked to chiral symmetry restoration in nuclear matter, and shed light to some parts of the QCD phase diagram.

At low energies the neutrino interacts with composite entities such as nucleons or nuclei. Given enough energy, the neutrino can actually begin to resolve the internal structure of the target: the neutrino can scatter off an individual quark inside the nucleon: DIS (deep inelastic scattering) and it manifests in the creation of a hadronic shower.

We will focus on the neutrino charged-current (CC) or neutral current (NC) inclusive reaction:

$$
v_{l}+A_{Z} \rightarrow l^{-}+X, \quad v_{l}+A_{Z} \rightarrow v_{l}+X .
$$

\footnotetext{
a e-mail: jmnieves@ific.uv.es
}

This is an Open Access article distributed under the terms of the Creative Commons Attribution License 4.0, which permits unrestricted use, distribution, and reproduction in any medium, provided the original work is properly cited. 
The double differential cross section, with respect to the outgoing lepton kinematical variables is given in the Laboratory (LAB) frame by

$$
\frac{d^{2} \sigma_{\nu_{l}}}{d \Omega\left(\hat{k}^{\prime}\right) d E_{l}^{\prime}}=\frac{\left|\vec{k}^{\prime}\right|}{|\vec{k}|} \frac{G_{F}^{2}}{4 \pi^{2} \eta} L_{\mu \sigma}^{(\nu)} W^{\mu \sigma}
$$

with $\vec{k}$ and $\vec{k}^{\prime}$ the LAB lepton momenta, $E_{l}^{\prime}$ the energy of the outgoing lepton, $G_{F}=1.1664$ $\times 10^{-11} \mathrm{MeV}^{-2}$, and $L$ and $W$ are the leptonic and hadronic tensors, respectively. Besides, $\eta=1(4)$ for $\mathrm{CC}(\mathrm{NC})$ processes. The leptonic tensor reads $\left(\epsilon_{0123}=+1\right)$

$$
L_{\mu \sigma}^{(\nu)}=L_{\mu \sigma}^{s}+\mathrm{i} L_{\mu \sigma}^{a}=k_{\mu}^{\prime} k_{\sigma}+k_{\sigma}^{\prime} k_{\mu}-g_{\mu \sigma} k \cdot k^{\prime}+\mathrm{i} \epsilon_{\mu \sigma \alpha \beta} k^{\prime \alpha} k^{\beta} .
$$

The hadronic tensor includes a collection of non-leptonic vertices and corresponds to the charged or neutral electroweak transitions of the target to all possible final states. It is completely determined by six independent, Lorentz scalar and real, structure functions. After contracting with the leptonic tensor, one obtains that for massless leptons ${ }^{1}$ only three of them, $F_{1,2,3}^{v}\left(x, q^{2}\right)$, are relevant, namely

$$
\frac{d^{2} \sigma_{v_{l}}}{d x d y}=\frac{G_{F}^{2} M E_{v}}{\eta \pi}\left\{\left(1-y-\frac{M x y}{2 E_{v}}\right) F_{2}^{v}+x y^{2} F_{1}^{v}+y(1-y / 2) x F_{3}^{v}\right\}
$$

with $E_{v}$ the incoming neutrino energy, $M$ the nucleon mass, $q=k-k^{\prime}, x=-q^{2} / 2 M q^{0}, y=q^{0} / E_{v}$. The cross section for the CC antineutrino induced nuclear reaction is easily obtained by i) changing the sign of the parity-violating term, proportional to $F_{3}$, in the differential cross section (this is because $L_{\mu \sigma}^{(\bar{v})}=L_{\sigma \mu}^{(\nu)}$ ), Eq. (4), and ii) using the appropriate weak current in the definition/computation of the hadron tensor. In the case of antineutrino NC driven processes, it is only needed to flip the sign of the term proportional to $F_{3}$ in the differential cross section, since the hadron NC is not affected.

The hadronic tensor is determined by the $W$ or $Z$ gauge boson selfenergy in the nuclear medium. To evaluate this latter object requires a theoretical scheme, where the relevant degrees of freedom and nuclear effects could be taken into account.

\section{High energy cross sections $E_{v} \gg 1-5 \mathrm{GeV}, Q^{2}=-q^{2} \gg 1 \mathrm{GeV}^{2}$}

This kinematic region is mostly out of the resonance production region and it is fully dominated by DIS processes. DIS offers an opportunity to probe the partonic structure of the nucleon both in its free state and when the nucleon is bound in a nucleus. Actually DIS has been extensively used to validate the standard model and probe nucleon structure. The structure of the nucleon is described in terms of parton distribution functions (PDFs) giving the longitudinal, transverse, and spin distributions of quarks within the nucleon. Neutrino scattering can play an important role in extraction of these fundamental PDFs since only neutrinos via the CC weak interaction can resolve the flavor of the nucleon's constituents: $v$ interacts with $d, s, \bar{u}$ and $\bar{c}$ while the $\bar{v}$ interacts with $u, c, \bar{d}$ and $\bar{s}$. High-statistics measurement of the nucleon's partonic structure, using neutrinos, could complement studies with electromagnetic probes.

In DIS processes, the neutrino scatters off a quark in the nucleon via CC or NC interactions producing a lepton in the final state, together with a hadronic shower. The Bjorken scaling variable, $x$ introduced in Eq. (4) plays an important role in deep inelastic neutrino scattering, where the quark can carry a portion of the incoming energy momentum of the struck target. The variable $y$ is related to the energy of the hadronic system.

\footnotetext{
${ }^{1}$ One should bear in mind that in the case of CC $v_{\tau}$ interactions, the $\tau$-lepton mass produces large differences [2].
} 

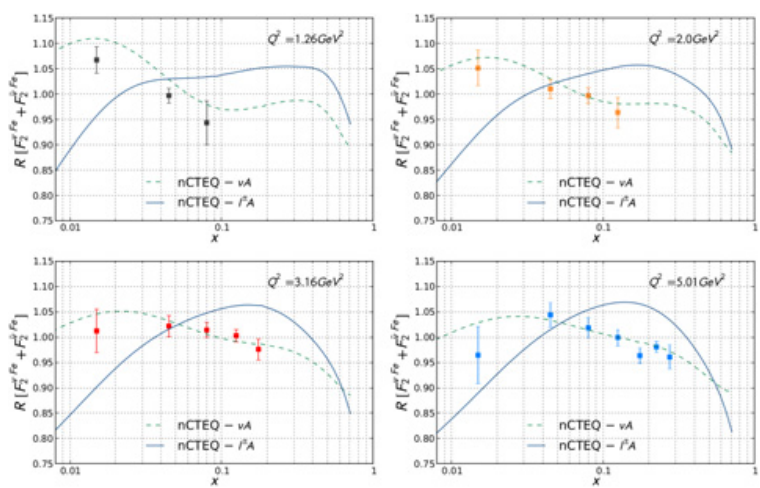

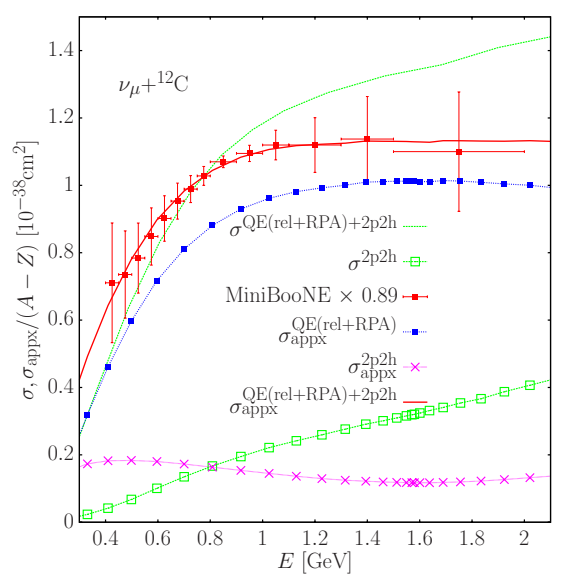

Figure 1. Left: Nuclear correction factor $R$ for the average $F_{2}$ structure function in $\mathrm{CC} v, \bar{v}$ Fe scattering at $Q^{2}=$ 1.26, 2.0, 3.16, and 5.01 $\mathrm{GeV}^{2}$ compared to the measured NuTeV points [6]. The dashed lines show the result of the nCTEQ [5] analysis of $\nu A$ (CHORUS [7], CCFR/NuTeV [8]) differential cross sections plotted in terms of the average $F_{2}^{\mathrm{Fe}}$ divided by the results obtained with the reference fit (free proton) PDFs. For comparison, the solid curve stands for the nCTEQ fit [5] to the charged-lepton data. Right: $v_{\mu}+{ }^{12} \mathrm{C}$ cross sections. Theoretical $(\sigma)$ and approximate $\left(\sigma_{a p p x}\right)$ CCQE-like integrated cross sections as a function of the $v$ energy (see Ref. [9] for further details). The MiniBooNE data [10] and errors (shape) have been re-scaled by a factor 0.9, as a result of the analysis carried out in Ref. [11]. All theoretical results have been obtained with the model of Refs. [12, 13] and $M_{A}=1.05 \mathrm{GeV}$.

Assuming the quark parton model, the structure functions $F_{i}\left(x, Q^{2}\right)$ in Eq. (4), are expressed in terms of the quark composition of the target. In the case of scattering off nucleons,

$$
F_{2}\left(x, Q^{2}\right)=2 \sum_{i=u, d, \ldots}\left[x q\left(x, Q^{2}\right)+x \bar{q}\left(x, Q^{2}\right)\right], \quad x F_{3}\left(x, Q^{2}\right)=2 \sum_{i=u, d, \ldots}\left[x q\left(x, Q^{2}\right)-x \bar{q}\left(x, Q^{2}\right)\right] .
$$

The struck quark carries a fraction $x$ of the nucleon's momentum, such that $x q(x \bar{q})$ is the probability of finding the quark (antiquark) with a given momentum fraction (PDFs). Besides,

$$
2 x F_{1}\left(x, Q^{2}\right)=\frac{\left(1+4 M^{2} x^{2} / Q^{2}\right) F_{2}\left(x, Q^{2}\right)}{1+R_{L}\left(x, Q^{2}\right)}
$$

where $R_{L}\left(x, Q^{2}\right)=\sigma_{L} / \sigma_{T}$ is the ratio of cross sections for scattering off longitudinally and transversely polarized exchange bosons. In the pursuit of precision measurements of neutrino oscillation parameters, large data samples and dedicated effort to minimize systematic errors have allowed charged lepton and neutrino experiments to independently isolate all six of the CC weak structure functions $F_{1}^{\nu N}\left(x, Q^{2}\right)$, $F_{1}^{\bar{\nu} N}\left(x, Q^{2}\right), F_{2}^{\nu N}\left(x, Q^{2}\right), F_{2}^{\bar{\nu} N}\left(x, Q^{2}\right), F_{3}^{\nu N}\left(x, Q^{2}\right), F_{3}^{\bar{\nu} N}\left(x, Q^{2}\right)$ over a wide range of $x$ and $Q^{2}$ values [4]. Taking differences and sums of these structure functions, specific PDFs in a given $x, Q^{2}$ bin can in turn be better isolated. Extracting this full set of structure functions relies on the $y$-variation of the structure function coefficients in the expression for the cross-section.

To achieve realistic descriptions of DIS processes, additional effects, like non-vanishing lepton masses, QCD corrections, heavy quark or nuclear and radiative corrections need also to be considered. These corrections are typically well understood and do not produce large uncertainties in the cross sections [2]. We illustrate this for the case of nuclear corrections in the left panels of Fig. 1. There, we see nuclear corrections turn out to be small, of the order of 5\%, and are successfully described by the nCTEQ analysis [5]. In general, there exist some tension between charged-lepton $\left(l^{ \pm} A\right)$ and the neutrino 
$(v A)$ data, as these plots suggest, and the size of the nuclear corrections extracted from neutrino data are smaller than those obtained from charged lepton scattering.

\section{Intermediate energy cross sections}

Below the DIS region, the neutrino scatters off nucleons rather than its constituent partons. We distinguish between inelastic and elastic or quasielastic processes.

\subsection{Inelastic processes}

Given enough energy, neutrinos can excite the struck nucleon to an excited state. In this case, the neutrino interaction produces a baryon resonance $N^{*}$. The baryon resonance quickly decays, most often to a nucleon and single pion final state. Resonances can also decay to multipion final states, and in general neutrino interactions in this energy range can also produce final states involving kaons and hyperons. DIS is also a source of multipion and kaon final states depending on the neutrino energy.

Because of the inherent complexity of reconstructing multiple pions final states, there are not many existing experimental measurements of this process. All of the existing measurements have been performed strictly using deuterium-filled bubble chambers.

The main reaction channels involving strange quarks are $K(\Delta S=1)$ and $\bar{K}(\Delta S=-1)$ production

$$
\begin{array}{ll}
v_{l} p \rightarrow l^{-} K^{+} p, & \bar{v}_{l} p \rightarrow l^{+} K^{-} p, \\
v_{l} n \rightarrow l^{-} K^{+} n, & \bar{v}_{l} n \rightarrow l^{+} K^{-} n, \\
v_{l} n \rightarrow l^{-} K^{0} p, & \bar{v}_{l} p \rightarrow l^{+} \bar{K}^{0} n,
\end{array}
$$

and $\mathrm{CC}$ and $\mathrm{NC}$ associated strangeness production $(\Delta S=0)$

$$
\begin{array}{lll}
v_{l} n \rightarrow l^{-} K^{+}\left(\Lambda, \Sigma^{0}\right), & \left(v_{l}, \bar{v}_{l}\right) p \rightarrow\left(v_{l}, \bar{v}_{l}\right) K^{+}\left(\Lambda, \Sigma^{0}\right), \\
v_{l} n \rightarrow l^{-} K^{0} \Sigma^{+}, & \left(v_{l}, \bar{v}_{l}\right) n \rightarrow\left(v_{l}, \bar{v}_{l}\right) K^{+} \Sigma^{-}, \\
v_{l} p \rightarrow l^{-} K^{+} \Sigma^{+}, & \left(v_{l}, \bar{v}_{l}\right) n \rightarrow\left(v_{l}, \bar{v}_{l}\right) K^{0}\left(\Lambda, \Sigma^{0}\right), \\
\bar{v}_{l} p \rightarrow l^{+} K^{+} \Sigma^{-}, & \left(v_{l}, \bar{v}_{l}\right) p \rightarrow\left(v_{l}, \bar{v}_{l}\right) K^{0} \Sigma^{+}, \\
\bar{v}_{l} p \rightarrow l^{+} K^{0}\left(\Lambda, \Sigma^{0}\right), & \\
\bar{v}_{l} n \rightarrow l^{+} K^{0} \Sigma^{-} . &
\end{array}
$$

Associated strangeness production is the dominant process but it has a high threshold because both a kaon and a hyperon are produced. This leaves single kaon production as the main source of kaons at low energies, in spite of being Cabibbo suppressed. These reactions typically have small cross sections because the kaon channels are not enhanced by any dominant resonance.

Weak pion production off nucleons cross sections grow to become one of the main reaction mechanisms for neutrinos of few-GeV energies. The first requirement to put neutrino induced pion production on nuclear targets on a firm ground is to have a realistic model at the nucleon level. Most of the models describe the pion production process by means of the weak excitation of the $\Delta(1232)$ resonance and its subsequent decay into $N \pi$, and in some occasions incorporate background terms. The major part of the models includes also the weak excitation of higher resonances as intermediate states. Vector form factors are fixed from helicity amplitudes extracted in the analysis of pion electroproduction data, while the axial couplings are obtained from PCAC [3]. Last years have witnessed an intense experimental and theoretical activity in this topic (see for instance Refs. [1-3] and references therein). Here, we will only make two remarks. The first one is that the Rein-Sehgal model [14], used by almost all Monte Carlo generators, provides a really poor description of the pion electroproduction data on 
protons $[15,16]$. Indeed, the model underestimates significantly the electron data, and it also reveals itself unsatisfactory in the axial sector at $q^{2}=0$, where the divergence of axial current can be related to the $\pi N$ amplitude by PCAC. Secondly, we would like to stress that, in sharp contrast to the previous reactions, weak pion production in dense matter is strongly affected by nuclear corrections, which might not be under control. Among these corrections, those that account for the interactions with the nucleons of the particles produced inside the nucleus on their way out are important. These corrections are commonly referred as final state interaction (FSI) effects. As example of the theoretical difficulties faced we refer the reader to the MiniBooNE flux-folded differential $d \sigma / d p_{\pi}$ cross section data in mineral oil reported in Ref. [17], which cannot be described by the well theoretically founded models of Refs. [18] and [19]. The latter approach is based in the model of Ref. [20] for weak pion production reaction off nucleons, which includes background non-resonant chiral terms and deuteron corrections [21], and it uses a Monte Carlo cascade model to account for FSI of the outgoing pion. The approach of Ref. [18] considers the weak excitation of several resonance contributions as intermediate states, and it implements a transport model to include FSI effects. Both models provide similar predictions for the MiniBooNE flux-folded differential $d \sigma / d p_{\pi}$ cross section, where FSI effects are clearly visible in the distribution. Because of the FSI some pions are absorbed, but other ones are scattered and lose to nucleons part of their energy. FSI is essential to fill the low momentum part of the distribution. On the other hand, the combined effect of pion quasi-elastic scattering and pion absorption through $\Delta$ excitation, depletes the $p_{\pi} \sim 250-450 \mathrm{MeV}$ region producing a distortion of the differential cross section shape that significantly worsens the description of the data. The artificial exclusion of the FSI effects leads to a better description of the $d \sigma / d p_{\pi}$ distribution. We refer the reader to Refs. $[3,18,19]$ for a more detailed discussion on this open problem.

\subsection{Quasielastic and elastic processes}

Below $2 \mathrm{GeV}$, neutrino-nucleus interactions are predominantly quasielastic $(\mathrm{QE})$, where the neutrino scatters off a nucleon bound in the nucleus. In the case of CC interactions at the nucleon level the possible reactions are $\nu_{l} n \rightarrow p l^{-}$and $\bar{v}_{l} p \rightarrow n l^{+}$, while for $\mathrm{NC}$, neutrinos elastically scatter from nucleons, $v N \rightarrow v N$. In these reactions nuclear effects are quite important $[1,3]$ and they need to be accounted for when determining the neutrino oscillation parameters. Indeed, uncertainties on these nuclear corrections are an important limitation in the oscillation analysis and a source of systematics.

As an example of the importance of the nuclear corrections, we will briefly describe here the so called MiniBooNE CCQE puzzle. This collaboration reported a CCQE cross section measurement [10] using a high-statistics sample of $v_{\mu}$ interactions on ${ }^{12} \mathrm{C}$. The size of the cross section was found to be unexpectedly large, and within the relativistic global Fermi gas model employed in the analysis of Ref. [10], a large nucleon axial mass of $M_{A}=1.35 \pm 0.17 \mathrm{GeV}$ was needed to describe the data. This value was in conflict with that extracted from CCQE measurements on deuterium and, to a lesser extent, hydrogen targets $M_{A}=1.016 \pm 0.026 \mathrm{GeV}$ [22], which is in excellent agreement with the pion electro-production result, $M_{A}=1.014 \pm 0.016 \mathrm{GeV}$, obtained from the nucleon axial radius [3, 23]. Furthermore, NOMAD also reported a small value of $M_{A}=1.05 \pm \pm 0.06 \mathrm{GeV}$ [24]. The MiniBooNE data-set (CCQE-like) accounted for events with no pions in the final state (events in which only one muon is detected), but Monte Carlo correcting for those cases where $\mathrm{CC} 1 \pi$ production was followed by pion absorption. It was customary to take for granted that most of those events could be attributed to the QE scattering of the weak probe on a nucleon. This choice discards pions coming off the nucleus, since they will give rise to additional leptons after their decay. A natural solution to this puzzle came from the incorporation of RPA nuclear corrections to the QE cross section [11, 12] and multi-nucleon effects $[11,13,25,26]$ (see right panel of Fig. 1). The latter effects account for events where the gauge boson is absorbed by two interacting nucleons (excitation of a $2 \mathrm{p} 2 \mathrm{~h}$ nuclear component). Up to re-scattering processes which could produce secondary pions, $2 \mathrm{p} 2 \mathrm{~h}$ events will give rise to only 
one muon in the final state, and thus they are included in the MiniBooNE data-set. Thus, they could be experimentally misidentified as QE events. Moreover, these events affect the reconstruction of the incoming $v$ energy [9] and the flux-unfolded cross section (Fig. 1). The same scheme [27] describes also the MiniBooNE CCQE-like antineutrino data sample of Ref. [28].

\section{Conclusions}

Nuclear effects lead to sizable corrections on neutrino nucleus cross sections at low $Q^{2}<1 \mathrm{GeV}^{2}$, which should be incorporated in neutrino event generators.

This research has been supported by the Spanish Ministerio de Economía y Competitividad under the contracts FIS2011-28853-C02-02, FIS2014-51948-C2-1-P, FIS2014-57026-REDT and SEV-2014-0398, by Generalitat Valenciana under contract PROMETEOII/2014/0068.

\section{References}

[1] J.G. Morfin, J. Nieves, J.T. Sobczyk, Adv.High Energy Phys. 2012, 934597 (2012)

[2] J. Formaggio, G. Zeller, Rev.Mod.Phys. 84, 1307 (2012)

[3] L. Alvarez-Ruso, Y. Hayato, J. Nieves, New J.Phys. 16, 075015 (2014)

[4] K.A. Olive et al. (Particle Data Group), Chin. Phys. C38, 090001 (2014)

[5] K. Kovarik, I. Schienbein, F.I. Olness, J.Y. Yu, C. Keppel, J.G. Morfin, J.F. Owens, T. Stavreva, Phys. Rev. Lett. 106, 122301 (2011)

[6] M. Tzanov et al. (NuTeV), Phys. Rev. D74, 012008 (2006)

[7] G. Onengut et al. (CHORUS), Phys. Lett. B632, 65 (2006)

[8] I. Schienbein, J.Y. Yu, C. Keppel, J.G. Morfin, F. Olness, J.F. Owens, Phys. Rev. D77, 054013 (2008)

[9] J. Nieves, F. Sanchez, I. Ruiz Simo, M. Vicente Vacas, Phys.Rev. D85, 113008 (2012)

[10] A. Aguilar-Arevalo et al. (MiniBooNE Collaboration), Phys.Rev. D81, 092005 (2010)

[11] J. Nieves, I. Ruiz Simo, M. Vicente Vacas, Phys.Lett. B707, 72 (2012)

[12] J. Nieves, J.E. Amaro, M. Valverde, Phys.Rev. C70, 055503 (2004)

[13] J. Nieves, I. Ruiz Simo, M. Vicente Vacas, Phys.Rev. C83, 045501 (2011)

[14] D. Rein, L.M. Sehgal, Annals Phys. 133, 79 (1981)

[15] K.M. Graczyk, J.T. Sobczyk, Phys.Rev. D77, 053001 (2008)

[16] T. Leitner, O. Buss, U. Mosel, L. Alvarez-Ruso, PoS NUFACT08, 009 (2008), 0809. 3986

[17] A. Aguilar-Arevalo et al. (MiniBooNE Collaboration), Phys.Rev. D83, 052009 (2011)

[18] O. Lalakulich, U. Mosel, Phys.Rev. C87, 014602 (2013)

[19] E. Hernandez, J. Nieves, M.J. Vicente Vacas, Phys.Rev. D87, 113009 (2013)

[20] E. Hernandez, J. Nieves, M. Valverde, Phys.Rev. D76, 033005 (2007)

[21] E. Hernandez, J. Nieves, M. Valverde, M. Vicente Vacas, Phys.Rev. D81, 085046 (2010)

[22] A. Bodek, S. Avvakumov, R. Bradford, H.S. Budd, Eur.Phys.J. C53, 349 (2008)

[23] V. Bernard, N. Kaiser, U.G. Meissner, Phys.Rev.Lett. 69, 1877 (1992)

[24] V. Lyubushkin et al. (NOMAD Collaboration), Eur.Phys.J. C63, 355 (2009)

[25] M. Martini, M. Ericson, G. Chanfray, J. Marteau, Phys.Rev. C80, 065501 (2009)

[26] M. Martini, M. Ericson, G. Chanfray, J. Marteau, Phys.Rev. C81, 045502 (2010)

[27] J. Nieves, I. Ruiz Simo, M. Vicente Vacas, Phys.Lett. B721, 90 (2013)

[28] A. Aguilar-Arevalo et al. (MiniBooNE Collaboration), Phys.Rev. D88, 032001 (2013) 